\title{
ESTRUCTURA POBLACIONAL DE DONAX STRIATUS (BIVALVIA, DONACIDAE) EN PLAYA LAS BALSAS, GIBARA, CUBA
}

\author{
Frank A. Ocaña Borrego ${ }^{1 *}$, Alejandro Fernández ${ }^{1}$, Alexis Silva ${ }^{2}$, Pedro A. González ${ }^{1}$ y Yamilet García ${ }^{2}$
}

\begin{abstract}
RESUMEN
Donax striatus Linné 1767 es una de las dos especies de almejas de playa que se encuentra en el Caribe. Pocas investigaciones se han centrado en describir aspectos sobre la estructura poblacional de esta especie. Una población de D. striatus fue muestreada mensualmente desde febrero del 2008 hasta enero del 2009 en playa Las Balsas, Gibara, Cuba. La población mostró fluctuaciones estacionales de la densidad, presentando los mayores valores en los meses de mayo y octubre del 2008 y enero del 2009. Se encontraron tres picos de reclutamiento: abril-mayo, agosto del 2008 y enero del 2009. Se observó una distribución estratificada por grupos de tallas: los reclutas fueron registrados en los estratos superiores de la playa y los adultos, fundamentalmente, en los estratos bajos. La mayor abundancia se localizó en el estrato intermedio. No hubo correlación entre los cambios mensuales de densidad con las temperaturas, ni con las precipitaciones, sin embargo, parece que los cambios en el régimen habitual del oleaje y la elevación del nivel medio del mar tienen influencia sobre el comportamiento de la abundancia de esta especie. Se propone una hipótesis que combina parámetros morfodinámicos para explicar la distribución vertical de los diferentes componentes de la población.
\end{abstract}

Palabras claves: Bivalvia, Donax, playas arenosas, estructura poblacional, Cuba.

\begin{abstract}
Donax striatus Linné 1767 is one of the two beach clam species that inhabits in the Caribbean. Few investigations have focused on describing aspects related with the population structure of this species. A population of D. striatus was surveyed from February 2008 to January 2009 in Las Balsas beach, Gibara, Cuba. The population showed seasonal fluctuations of the density presenting the higher values on May and October 2008 and January 2009. There were peaks of recruitment: April-May, August 2008 and January 2009. A stratified distribution by size classes was observed: recruits were found in the higher strata of the beach while adults occur mainly in the lowest strata. The higher abundance was found in the middle strata. There was no correlation between monthly changes in density and temperatures, nor with the rainfall however, changes in the normal wave's regimen and mid sea level rise seems to have some influence over the behaviour of this species abundance. A hypothesis that combines morphodynamics parameters to explain the across shore distribution of the components of the population, is proposed.
\end{abstract}

Keywords: Bivalvia, Donax, sandy beaches, population structure, Cuba.

\section{INTRODUCCIÓN}

Las playas arenosas están consideradas entre los ambientes más dinámicos que existen, siendo, de todos los ecosistemas marinos, los más controlados por factores físicos (McLachlan, 1990). A simple vista, las playas parecen lugares carentes de vida, pero enterrados en los sedimentos se pueden encontrar diversos organismos. Los moluscos bivalvos del género Donax son un componente importante de la macrofauna de las playas arenosas en zonas templadas, tropicales y subtropicales (Ansell, 1983). Estos son los organismos dominantes en este tipo de

1 Centro de Inv. y Serv. Amb. y Tec. 18 s/n. esq. a Maceo. Rpto., "El Llano”, Holguín, Cuba. franko@cisat.cu*

2 Museo de Historia Natural "Joaquín Fernández de la Vara", Gibara, Holguín, Cuba.

Recibido 22-III-2009

Aceptado 27-I-2010

DOI: http://dx.doi.org/10.15359/revmar.2.2 
ambiente, tal éxito puede ser atribuido a que presentan una alta capacidad de coordinar rápidamente los movimientos de enterramiento y migración como respuesta a la acción del oleaje, las mareas y las corrientes (Loesch, 1957; Turner y Belding, 1957; Wade, 1967a; Tiffany, 1971; Trueman, 1971; McLachlan et al. 1979; Leber, 1982; Ansell, 1983; Ellers, 1995).

Las especies del género Donax han atraído la atención de numerosos investigadores, debido a los atributos antes mencionados, a la accesibilidad a su hábitat y a las altas fluctuaciones de sus poblaciones. Los cambios estacionales de la abundancia pueden variar entre unos pocos individuos en un año hasta miles en el próximo, este fenómeno fue documentado por Coe (1953), que lo denominó población resurgente.

Donax striatus Linné 1767 es una de las dos especies de almejas de playa que se encuentra en el Caribe. Su rango de distribución comienza por Gibara, Cuba, en el norte hasta Areia Branca en Brasil (Wade, 1967b). Pocas investigaciones se han centrado en describir aspectos sobre la estructura poblacional de esta especie y en la literatura no se ha encontrado ninguna llevada a cabo en las playas de Cuba. El objetivo del presente estudio es describir la estructura poblacional de $D$. striatus en playa Las Balsas, Gibara, Cuba.

\section{MATERIALES Y MÉTODOS}

Las Balsas es una playa protegida que se ubica hacia el sur de la bahía de Gibara en Holguín, Cuba (Fig. 1). Tiene una longitud de $1600 \mathrm{~m}$, con un ancho promedio de la cara de la playa de $8.75 \mathrm{~m}$, sus arenas presentan una granulometría media y son de origen terrígeno. La playa limita al oeste con la desembocadura del río Cacoyugüin y al este con la desembocadura del río Gibara.

El muestreo se realizó mensualmente desde febrero del 2008 hasta enero del 2009, durante la marea baja. Tres estaciones fijas

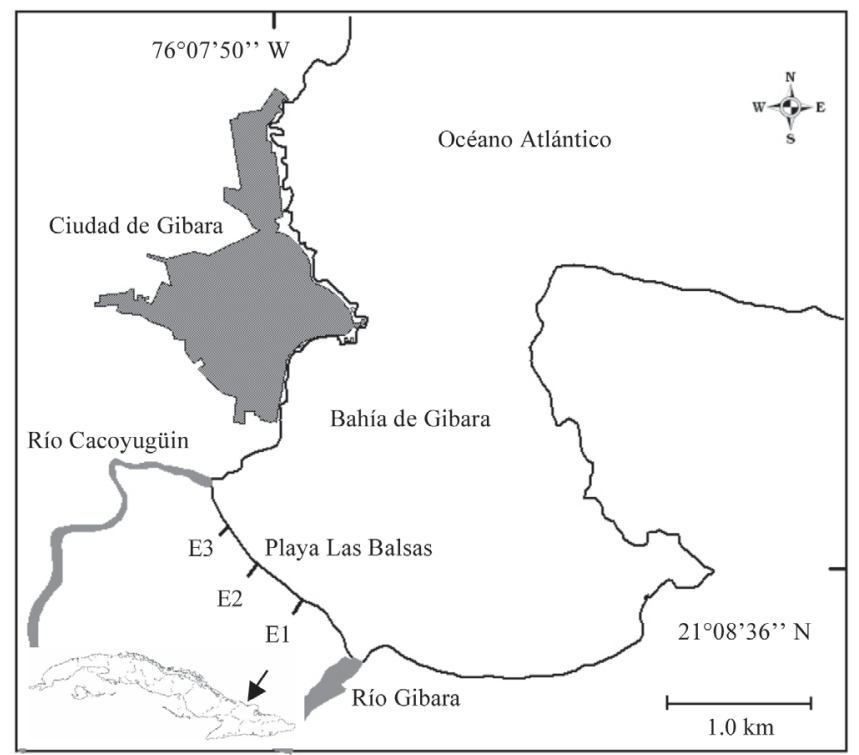

Fig. 1. Ubicación geográfica de playa Las Balsas y representación de las estaciones de muestreo (E1, E2 y E3)

Fig. 1. Geographic location of Las Balsas beach and representation of sampling stations (E1, E2 y E3) 
fueron seleccionadas (E1, E2 y E3). La obtención de las muestras se realizó empleando un cilindro de PVC, con un diámetro de $18 \mathrm{~cm}$ y una altura de $30 \mathrm{~cm}$, introduciéndolo en el sustrato arenoso hasta una profundidad de $20 \mathrm{~cm}$ (Unidad de muestreo, UM). En cada estación se tomaron muestras en estratos separados a intervalos de $1 \mathrm{~m}$ perpendicular a la línea de costa, a partir del punto de inflexión o escalón de la playa en la zona intermareal (P1), hasta el límite superior de la zona de barrido del oleaje (P5), en algunas ocasiones los individuos se encontraron más arriba de P5, pero estos datos no fueron empleados para comparar la distribución por estratos. En cada estrato se extrajeron tres cilindros de $0.025 \mathrm{~m}^{2}$, separados cada $1 \mathrm{~m}$. El sedimento fue cribado en un tamiz de 1 $\mathrm{mm}$ de abertura de malla. Los individuos de $D$. striatus se introdujeron en bolsas plásticas y se congelaron.

En el laboratorio, a cada individuo se le determinó la longitud anteroposterior con el empleo de un Vernier digital, con una precisión de $0.01 \mathrm{~mm}$. Los individuos fueron agrupados en intervalos de clases de $1 \mathrm{~mm}$, para elaborar los histogramas de distribución de tallas en cada mes. La abundancia (ind $/ \mathrm{m}^{2}$ ) se determinó mensualmente. Los especímenes también se categorizaron en tres grupos: reclutas (menores de $5 \mathrm{~mm}$ ), juveniles $(5 \mathrm{~mm}-15 \mathrm{~mm}$ ) y adultos (mayores de $15 \mathrm{~mm}$ ). Para evaluar si existe asociación entre las clases de tallas y los estratos, se aplicó una prueba Chi-cuadrado $\left(\mathrm{X}^{2}\right)$. Para determinar si existen diferencias en la densidad por meses, se realizó una prueba de Kruskal-Wallis (K-W).

Datos de temperatura media $\left({ }^{\circ} \mathrm{C}\right) \mathrm{y}$ precipitaciones media $(\mathrm{mm})$, aportados por la Estación Velasco del Centro Meteorológico Provincial, fueron empleados para determinar la influencia de estas variables abióticas sobre la densidad poblacional a través de una prueba de correlación de rangos de Spearman $\left(\mathrm{r}_{\mathrm{s}}\right)$. Todas las pruebas estadísticas fueron llevadas a cabo empleando el paquete STATISTICA 6.1 (StatSoft Inc., 1995). Las diferencias estadísticas fueron consideradas con un nivel de significación de 95\% $(\alpha=0.05)$ (Zar, 1999) para K-W y r. . Para $X^{2}$, el nivel de significación empleado fue de $99 \%$.

\section{RESULTADOS}

La población de $D$. striatus en playa Las Balsas presenta fluctuaciones en su composición a lo largo de todo el año (Fig. 2), manifestándose tres picos bien

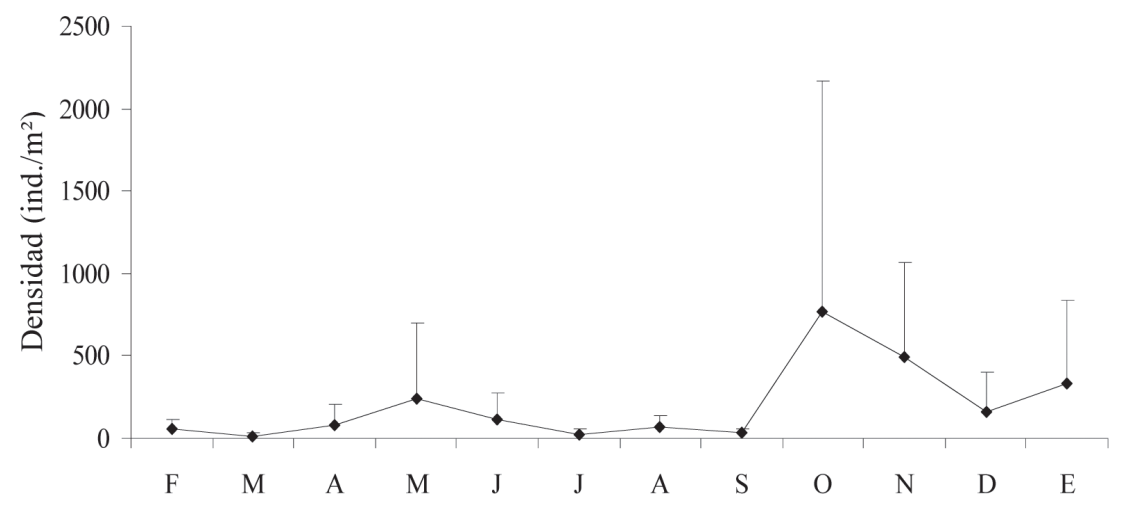

Fig. 2. Densidad poblacional de D. striatus (media+D.E.) en playa Las Balsas

Fig. 2. Population density of D. striatus (mean+S.D.) at Las Balsas beach 
Cuadro 1. Valores de densidad mensual (ind $/ \mathrm{m}^{2}$ ) de D. striatus en playa Las Balsas (UM: número de unidades de muestreo; D. E.: desviación estándar)

Table 1. Monthly density values (ind $/ \mathrm{m}^{2}$ ) of D. striatus at Las Balsas beach (UM: number of sample units; S. D.: standard deviation)

\begin{tabular}{lccccc}
\hline \multicolumn{1}{c}{ Meses } & UM & Mínimo & Máximo & Media & D.E. \\
\hline Febrero & 27 & 0.00 & 120.00 & 53.33 & 56.57 \\
Marzo & 36 & 0.00 & 40.00 & 17.10 & 12.85 \\
Abril & 42 & 0.00 & 453.30 & 77.02 & 130.21 \\
Mayo & 30 & 0.00 & 1480.00 & 238.67 & 456.70 \\
Junio & 30 & 0.00 & 520.00 & 112.01 & 160.40 \\
Julio & 36 & 0.00 & 106.70 & 25.56 & 29.80 \\
Agosto & 27 & 0.00 & 200.00 & 65.19 & 77.02 \\
Septiembre & 30 & 0.00 & 80.00 & 34.67 & 24.50 \\
Octubre & 30 & 0.00 & 3866.70 & 770.67 & 1401.32 \\
Noviembre & 54 & 13.30 & 1840.00 & 497.79 & 567.46 \\
Diciembre & 51 & 13.30 & 760.00 & 163.12 & 239.66 \\
Enero 2009 & 48 & 13.30 & 1666.70 & 328.32 & 513.38 \\
\hline
\end{tabular}

definidos en la abundancia en los meses de mayo y octubre del 2008 y en enero del 2009. Los meses de marzo, julio y septiembre fueron donde se presentaron los valores más bajos de densidad, con menos de $35 \mathrm{ind} / \mathrm{m}^{2}$ (Cuadro 1 ).

Los valores menores de temperaturas fueron registrados en enero del 2009 $\left(23.0^{\circ} \mathrm{C}\right)$ y los más elevados en el mes de julio, con $28.4^{\circ} \mathrm{C}$. Las precipitaciones presentaron altas variaciones, registrándose los valores mínimos $(13.0 \mathrm{~mm})$ en el mes de julio y los máximos en octubre (186.8 mm) (Fig. 3). La densidad presentó dife- rencias significativas entre los meses, sin embargo, no tuvo correlación con la temperatura y las precipitaciones (Cuadro 2).

La figura 4 muestra la distribución de frecuencias de tallas durante el período muestreado. La menor talla registrada fue de $2.74 \mathrm{~mm}$ y la longitud máxima alcanzada fue de $30.72 \mathrm{~mm}$. El mayor porcentaje de individuos, durante todo el período de estudio, estuvo representado por individuos con tallas entre $5 \mathrm{~mm}$ y $20 \mathrm{~mm}$. La proporción de individuos por encima de los $20 \mathrm{~mm}$ de longitud fue muy baja, encontrándose la mayor inci-

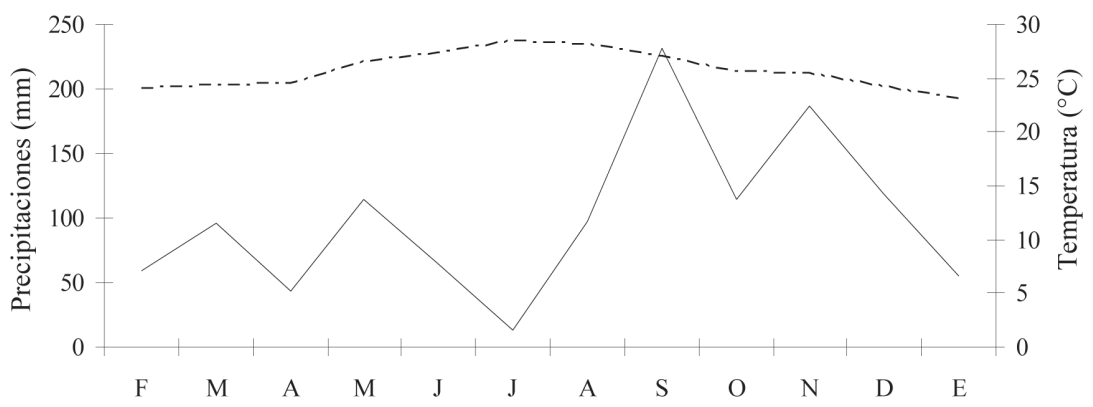

Fig. 3. Distribución mensual de las temperaturas (línea de puntos) y las precipitaciones (línea continua) registradas en la Estación Velasco

Fig. 3. Monthly distribution of temperatures (dotted line) and rainfall (continued line) registered at Velasco meteorological station 
Cuadro 2. Resultados de los análisis estadísticos empleados para determinar las relaciones entre la densidad de D. striatus con los meses, las temperaturas promedio y las precipitaciones promedio. n.s.: no hay diferencias, *: diferencias significativas

Table 2. Results of the statistical analysis employed to determine the relations among density of $D$. striatus with months, mean temperatures and mean rainfall. n.s.: no differences, *: significant differences

\begin{tabular}{lcrc}
\hline \multicolumn{1}{c}{ Factores } & Prueba estadística & Valor & $\boldsymbol{P}$ \\
\hline Meses & $\mathrm{K}-\mathrm{W}$ & 34.34 & $0.0003 *$ \\
Temperaturas & $\mathrm{r}_{\mathrm{s}}$ & -0.249 & 0.4705 n.s. \\
Precipitaciones & $\mathrm{r}_{\mathrm{s}}$ & 0.301 & 0.3423 n.s. \\
\hline
\end{tabular}

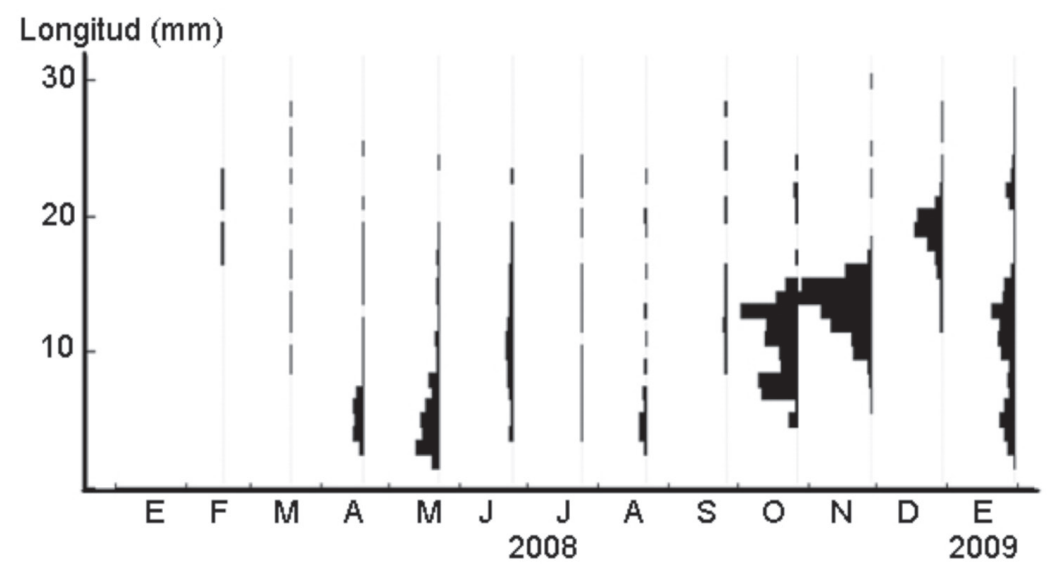

Fig. 4. Distribución mensual de las frecuencias de clases de tallas de D. striatus en playa Las Balsas desde febrero del 2008 hasta enero del 2009

Fig. 4. Monthly length-frequency distribution of $D$. striatus at Las Balsas beach from February 2008 to January 2009

dencia en diciembre del 2008 y en enero del 2009. Se observan tres picos de reclutamiento: abril-mayo, agosto del 2008 y enero del 2009. En el mes de octubre se registró una alta abundancia de individuos, pero la mayoría presentaron tallas entre $5 \mathrm{~mm}$ y $15 \mathrm{~mm}$.

En la figura 5 se muestra la distribución de reclutas, juveniles y adultos a lo largo de los estratos. Existe una asociación muy significativa $\left(\mathrm{X}^{2}=288.72 ;\right.$ g.l $l=8 ; P=$ $0.0000)$ entre la composición por tallas y la distribución de los individuos en los diferentes estratos de la playa. Los reclutas se distribuyen fundamentalmente a partir del estrato 3 , mientras los juveniles y adultos se pueden encontrar a través de toda la playa, pero con una preferencia por los estratos 2 , 3 y 4 (Cuadro 3). En el estrato 1, la mayor proporción de individuos que se encontró durante el estudio fue de adultos. Se debe precisar que los individuos mayores de 20 $\mathrm{mm}$ se observaron solo en los estratos 1 y 2 a lo largo de todo el período, excepto en el mes de diciembre que se registraron también en los estratos superiores. El estrato 3 fue el que presentó el mayor número de individuos de las tres clases de tallas. 
Cuadro 3. Tabla de contingencia* (clases de tallas $\mathrm{x}$ estratos) para determinar la asociación de la distribución transversal de $D$. striatus. Reclutas: $<5 \mathrm{~mm}$, Juveniles: 5 mm-15 mm, Adultos: > 15 mm, P1-P5: estratos

Table 3. Contingency table* (size classes $\mathrm{x}$ strata) to determine the association of the across shore distribution of $D$. striatus. Recruits: $<5$ mm, Youth: 5 mm-15 mm, Adults: $>15 \mathrm{~mm}, \mathrm{P1}$-P5: strata

\begin{tabular}{lllllll}
\hline & \multicolumn{1}{r}{ P1 } & \multicolumn{1}{c}{ P2 } & \multicolumn{1}{c}{ P3 } & \multicolumn{1}{c}{ P4 } & \multicolumn{1}{c}{ P5 } & \multicolumn{1}{c}{ Total } \\
\hline Reclutas & 0 & 6 & 105 & 18 & 12 & 141 \\
& $0.00 \%$ & $0.29 \%$ & $4.99 \%$ & $0.86 \%$ & $0.57 \%$ & $6.70 \%$ \\
Juveniles & 29 & 194 & 949 & 210 & 53 & 1435 \\
& $1.38 \%$ & $9.22 \%$ & $45.13 \%$ & $9.99 \%$ & $2.52 \%$ & $68.24 \%$ \\
Adultos & 62 & 78 & 182 & 109 & 96 & 527 \\
& $2.95 \%$ & $3.71 \%$ & $8.65 \%$ & $5.18 \%$ & $4.56 \%$ & $25.06 \%$ \\
Total & 91 & 278 & 1236 & 337 & 161 & 2103 \\
& $4.33 \%$ & $13.22 \%$ & $58.77 \%$ & $16.02 \%$ & $7.66 \%$ & $100.00 \%$ \\
\hline
\end{tabular}

* - Datos acumulados, * - Cumulative data.

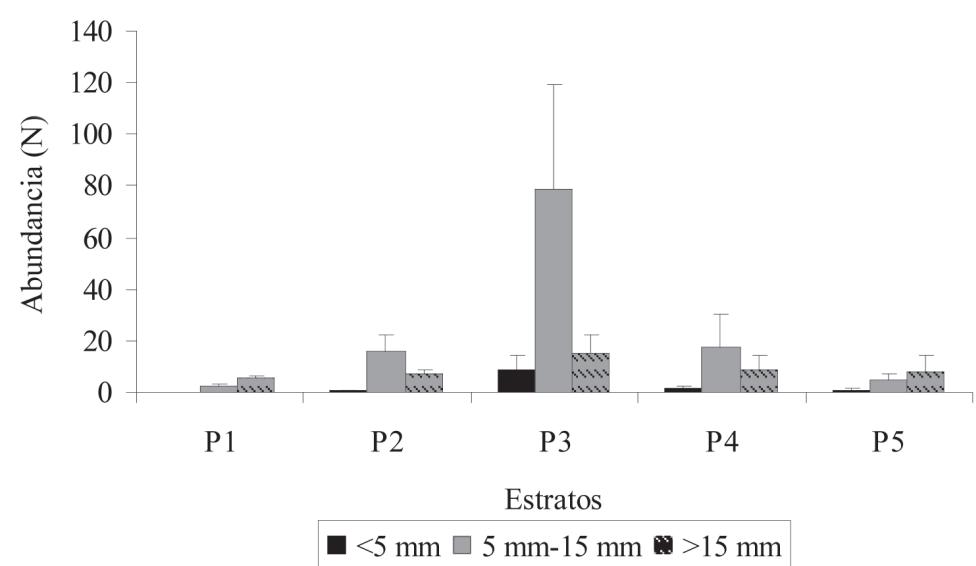

Fig. 5. Distribución espacial de la abundancia (media+ES) de D. striatus perpendicular a la costa por clases de tallas (reclutas: $<5 \mathrm{~mm}$, juveniles: $5 \mathrm{~mm}-15 \mathrm{~mm}$, adultos: $>15 \mathrm{~mm}$ ) y estratos (P1-P5) Fig. 5. Across shore spatial distribution of abundance (mean $+\mathrm{SE}$ ) of $D$. striatus by population components (recruits: $<5 \mathrm{~mm}$, youth: $5 \mathrm{~mm}-15 \mathrm{~mm}$, adults: $>15 \mathrm{~mm}$ ) and strata (P1-P5

\section{DISCUSIÓN}

Los cambios estacionales en la densidad de la población de D. striatus son similares a los que ocurren con otras especies del género en diferentes partes del mundo. Estas especies están en general sujetas a fuertes fluctuaciones naturales episódicas de su abundancia, debidas, fundamentalmente, a una alta variabilidad del reclutamiento y a una mortalidad masiva ocasional (Le Moal, 1993; Defeo y Alava, 1995).

Coe (1955), monitoreando una población de D. gouldii en California, determinó que esta llegó a alcanzar una densidad 
de $20000 \mathrm{ind} / \mathrm{m}^{2}$ en una franja de arena entre 3 y 5 metros de ancho, y en el año precedente en la misma localidad detectó una densidad de solo $1 \mathrm{ind} / \mathrm{m}^{2}$. Patrones similares de distribución estacional fueron observados por Mikkelsen (1981) para $D$. variabilis, por Zeichen et al. (2002) para D. trunculus y por Delgado et al. (2003) para D. striatus en Venezuela. En el caso particular de las especies $D$. striatus y $D$. denticulatus se han observado variaciones en las densidades poblacionales, cuando se comparan diferentes localidades e incluso dentro de una misma localidad en distintas épocas del año (McLachlan et al. 1996).

Estos cambios estacionales en la abundancia han sido tratados de explicar por la influencia de factores abióticos y bióticos. En Uruguay se encontró una correlación significativa entre el esfuerzo pesquero y la abundancia de D. hanleyanus. Los picos de densidad de esta especie se observaron cuando disminuyó o se eliminó el esfuerzo pesquero ejercido sobre el bivalvo simpátrico Mesodesma mactroides, sugiriendo una potencial interdependencia ecológica entre ambas especies (Defeo y Alava, 1995). En otra investigación con D. hanleyanus se observó relación entre la abundancia de esta especie, la intensidad del oleaje y la abundancia de otro bivalvo, Buccinanops duartei (Gil y Thomé, 2001). Estos mismos autores no encontraron evidencia de la influencia de las temperaturas y las precipitaciones. Cardoso y Veloso (2003) también observaron una correlación entre la abundancia de D. hanleyanus y el decápodo Emerita brasiliensis, pero no hubo evidencia de relación en el tiempo entre el número de individuos y las características morfodinámicas de la playa.

Los cambios de densidad observados en la población de $D$. striatus de playa Las
Balsas no parecen estar influenciados por las temperaturas y las precipitaciones, aunque no se cuenta con datos tomados in situ de la temperatura superficial del agua y de la salinidad. Un hecho interesante es que en días previos a los muestreos realizados en marzo y septiembre ocurrieron fenómenos de sobreelevación del nivel del mar, con la ocurrencia de oleajes fuertes, que sobrepasaron $1.5 \mathrm{~m}$ de altura, siendo más intensos en el mes de septiembre, debido al paso de la tormenta tropical Hannah y del huracán Ike. En la figura 2 se observa que en estos dos meses, los valores de abundancia fueron muy bajos y en los dos meses siguientes, la abundancia aumentó considerablemente. Después del paso del fenómeno "El Niño", se observaron aumentos importantes de la densidad poblacional en D. peruvianus en Perú (Arntz et al. 1987) y de $D$. dentifer en el pacífico colombiano (Riascos y Urban, 2002). Al parecer, los cambios en el régimen habitual del oleaje y la elevación del nivel medio del mar tienen influencia sobre el comportamiento de la abundancia de estas especies.

Se descarta la posible relación entre la abundancia de este bivalvo con la de otros organismos, pues esta especie es dominante y la diversidad es baja en este ambiente. El decápodo Hippa testudinaria está presente en el área, pero solo se cuantificaron cinco individuos durante todo el período de estudio. No se observaron números considerables de otros moluscos o de aves que pudieran ser depredadoras. Tampoco se cuantificó la abundancia del isópodo Eurydice sp., especie que comparte el mismo hábitat. La población de $D$. striatus es objeto de una pesquería recreativa por parte de la comunidad local, pero las capturas no son considerables y los niveles no manifiestan asociación con 
una estación determinada del año (Ocaña y Fernández en preparación).

D. striatus mostró un reclutamiento casi continuo durante el período de estudio. Resultados similares fueron observados para D. denticulatus por Wade (1968), Sastre (1984) y Vélez et al. (1985), sin embargo, para esta especie, Marcano et al. (2003) observaron en otra población que las distribuciones mensuales de longitud fueron bimodales y la proporción de bivalvos con tallas inferiores a $7 \mathrm{~mm}$ de longitud fue escasa, presentándose la mayor incidencia en octubre y noviembre. Rocha-Barreira et al. (2002) encontraron individuos de $D$. striatus menores de 6 $\mathrm{mm}$ durante todo el año, aunque la baja frecuencia de tallas mayores sugirió que estos reclutas provenían de áreas adyacentes a la playa estudiada.

El máximo reclutamiento encontrado en los meses de abril y mayo para la población de playa Las Balsas coincide, estacionalmente, con los resultados observados por Aguirre y Mendo (2008) para $D$. obesulus y por Wade (1968) para $D$. denticulatus. Wilson (1999) también encontró en $D$. variabilis, un reclutamiento continuo durante varios meses, con dos picos. En D. trunculus se observan distintos comportamientos de reclutamiento (Zeichen et al. 2002), no pudiéndose constatar un patrón uniforme. El hecho de no haber encontrado individuos menores de $5 \mathrm{~mm}$ en el mes de noviembre sugiere que ocurrió un rápido crecimiento de los reclutas en este mes, tal vez los efectos del huracán Ike ocasionaron un aumento en el nivel de alimento disponible posterior a su paso por las costas de Gibara.

Gil y Thomé (2004) encontraron que $D$. hanleyanus presenta una actividad reproductiva durante todo el año, de acuerdo con análisis histológicos realizados, sin embargo, estudiando el crecimiento de esta misma población, Gil y Thomé (2000a) observaron dos picos en el reclutamiento. De los resultados obtenidos en la población de Las Balsas y la revisión de otros trabajos con diferentes especies del género Donax en diferentes latitudes, no se puede evidenciar un patrón claro en cuanto al asentamiento de las nuevas progenies. Ansell (1983) mostró que las especies del género Donax presentan patrones diferenciales de reclutamiento, incluso dentro de una misma especie, estos patrones pueden cambiar entre zonas geográficas y entre años. Algunas de las causas relacionadas con este fenómeno pueden ser atribuidas a cambios en las temperaturas (Vélez et al. 1985) y a la disponibilidad de alimento.

Otro de los hallazgos sorprendentes, con respecto al género Donax, es que sus poblaciones manifiestan patrones de zonación correspondientes con las diferentes clases de tallas. Los juveniles tienden a asentarse en diferentes niveles antes de migrar hacia la zona de los adultos (McLachlan et al. 1996). La población estudiada en playa Las Balsas muestra un patrón definido de zonación por talla, con una distribución en forma de campana, a través de la zona de barrido del oleaje, existiendo la mayor abundancia de individuos de todas las clases de tallas en un estrato intermedio; así como los adultos se ubican preferentemente en la parte baja de la anteplaya y los reclutas se disponen en los estratos más altos. Comportamientos semejantes han sido observados en $D$. denticulatus (Wade, 1967a; Etchevers, 1975; Marcano et al. 2003), D. faba (Alagarswami, 1966), D. trunculus y D. vittatus (Ansell y Lagardére, 1980) y en $D$. hanleyanus (Gil y Thomé, 2000b). 
En otras especies de donácidos se ha documentado la segregación espacial entre los diferentes componentes de las poblaciones, como por ejemplo, entre adultos y reclutas. Este fenómeno ha sido tratado de explicar por distintas causas que pueden incluir la interacción de factores físicos y biológicos, que actúan en diferentes escalas espacio-temporales (Defeo y McLachlan, 2005). Entre los factores abióticos se pueden mencionar: las diferencias de temperatura entre los estratos de la zona intermareal y en la zona submareal (Bayed y Guillou, 1985), la cobertura de macroalgas arribadas a la playa (Soares et al. 1996, 1998), la granulometría (Mazé y Laborda, 1988) y la pendiente de la anteplaya (Cardoso y Veloso, 2003).

Desde el punto de vista de los factores bióticos, se sugiere en varios estudios que la segregación por tallas puede estar modulada por la competencia interespecífica (Marcano et al. 2003; Dugan et al. 2004), que puede ser una forma de evitar la depredación (Ansell y Lagardére, 1980; Leber, 1982) o es el resultado de la competencia por el espacio y el alimento o por el filtrado de las larvas por los adultos durante el proceso de alimentación (Defeo y McLachlan, 2005). Gaspar et al. (2002) también sugieren que en las poblaciones que se distribuyen en la zona submareal a diferentes profundidades, el asentamiento de los reclutas en la zona de rompiente del oleaje se debe a una mayor disponibilidad de alimentos. Gil y Thomé (2000b) relacionan la existencia de los individuos mayores en la zona más dinámica de barrido del oleaje con el grosor de la concha, así los animales más pequeños se encuentran en la zona superior, donde el dinamismo es menos estresante.

Al parecer, la distribución vertical por tallas descrita para las especies de Donax es un fenómeno que se manifiesta por causas diferentes en dependencia de la especie en cuestión y del espacio geográfico que estas ocupen. Schoeman et al. (2003) mostraron que los agrupamientos verticales sobre la anteplaya pueden desarrollarse en respuesta a algunos procesos, que actúan a una escala espacial cercana a $3 \mathrm{~m}$.

$D$. striatus es una de las especies menos estudiadas del género. El primer comentario sobre la zonación de esta especie fue realizado por Wade (1967b), pero solo bajo criterios de pocas observaciones. Para llegar a establecer los patrones de distribución se necesitan estudios con muestreos intensivos (Defeo y McLachlan, 2005).

A lo largo de un año, la población de playa Las Balsas mostró el mismo patrón de distribución en la zona de barrido del oleaje. Muestreos adicionales hacia la zona submareal registraron la presencia de algunos individuos fundamentalmente adultos, lo que demuestra que esta especie es capaz de migrar. Para explicar las observaciones sobre la distribución vertical de los individuos de la población se propone la siguiente hipótesis: los componentes de la población (reclutas, juveniles y adultos) muestran preferencia por un microhábitat. Los reclutas se asientan en la parte superior de barrido traídos por las olas, encontrando una zona donde el sedimento es más fino, la turbidez es menor y el dinamismo es menos estresante, aprovechando más energía en procesos de desarrollo que de mantenimiento. A medida que crecen ocupan la zona intermedia, donde las condiciones de la granulometría y el oleaje son óptimas, permitiéndoles un enterramiento fácil y un constante suministro de alimento y oxígeno. Luego, cuando alcanzan mayores tallas, son más capaces de resistir el embate del oleaje, 
coordinan rápidamente el enterramiento en un sedimento más grueso y evitan la competencia con los otros grupos (reclutas y juveniles) de la población.

La alta desviación estándar de los valores de densidad presentados en la figura 2 sugiere que esta especie tiene una distribución agrupada. Esta playa es micromareal, por lo que el cinturón de distribución de $D$. striatus es pequeño, los cambios en la estructura vertical de los individuos presentan variaciones a una escala de muy poca distancia siguiendo el gradiente de la anteplaya, donde la granulometría, la turbidez y la compactación del sedimento van disminuyendo desde los estratos superiores hasta el punto inferior de barrido, presentándose las condiciones ambientales óptimas en el centro de este gradiente, lo que condiciona la distribución unimodal de esta población.

\section{AGRADECIMIENTOS}

Agradecemos la colaboración de Dayanis Matos, por su apoyo en el procesamiento de las muestras y a Marko Herrmann, por facilitarnos gran parte de la literatura.

\section{BIBLIOGRAFÍA}

Aguirre, A. \& Mendo, J. (2008). Crecimiento y producción de Donax obesulus Reeve, 1854 (Bivalvia: Donacidae) en playa Sarapampa, Asia, Lima. Ecol. Apl., 7, 63-70.

Alagarswami, K. (1966). Studies on some aspects of biology of the wedge-clam Donax faba from Mandapam coast in the Gulf of Mannar. J. Mar. Biol. Assoc. India, 8, 56-75.

Ansell, A. D. \& Lagardére, F. (1980). Observations on the biology of Donax trunculus and Donax vittatus at Ile
d'Oléron (French Atlantic coast). Mar. Biol., 57, 287-300.

Ansell, A. D. (1983). The biology of the genus Donax. En A. McLachlan \& T. Erasmus (Eds.), Sandy beaches as ecosystems (pp. 607-636). The Hague: W. Junk.

Arntz, W. E., Brey, T. J., Tarazona, A. \& Robles, J. (1987). Changes in the structure of Shallow sandy beach community in Peru during an El Niño event. South African J. Mar. Sci., 5, 645-658.

Bayed, A. \& Guillou, J. (1985). Contribution à l'étude des populations du genere Donax: La population de D. trunculus L. (Mollusca, Bivalvia) de Mehdia (Maroc). Ann. l'Inst. Océanograph., 61, 139-147.

Cardoso, R. S. \& Veloso, V. G. (2003). Population dynamics and secondary production of the wedge clam Donax hanleyanus (Bivalvia: Donacidae) on a high-energy, subtropical beach of Brazil. Mar. Biol., 142, 153-162.

Coe, W. R. (1953). Resurgent populations of littoral marine invertebrates and their dependence on ocean currents and tidal currents. Ecol., 34, 225-229.

Coe, W. R. (1955). Ecology of the bean clam Donax gouldii on the coast of southern California. Ecol., 36, 512-514.

Defeo, O. \& de Alava, A. (1995). Effects of human activities on long-term trends in sandy beach populations: the wedge clam Donax hanleyanus in Uruguay. Mar. Ecol. Prog. Ser., 123, 73-82.

Defeo, O. \& McLachlan, A. (2005). Patterns, processes and regulatory mechanisms in sandy beach macrofauna: a multi-scale analysis. Mar. Ecol. Prog. Ser., 295, 1-20.

Delgado, J. G., Godoy, A. R., Reverol, Y. M. \& Severeyn, H. J. (2003). Fluctuaciones poblacionales de los moluscos bivalvos Donax striatus (Linné, 1767) y Tivela 
mactroides (Born, 1778) en dos playas arenosas de Venezuela. Biol. Venez., 23 (1), 33-35.

Dugan, J. E., Jaramillo, E., Hubbard, D. M., Contreras, H. \& Duarte, C. (2004). Competitive interactions in macroinfaunal animals of exposed sandy beaches. Oecol., 139, 630-640.

Ellers, O. (1995). Behavior control of swash-riding in the clam Donax variabilis. Biol. Bull., 189, 120-127.

Etchevers, S. (1975). Notas ecológicas y evaluación de la población de chipichipi Donax denticulatus (Linné, 1758) (Bivalvia-Donacidae) en la playa de La Restinga, Isla Margarita, Venezuela. En Memorias II Simposio Latinoamericano de Oceanografía Biológica (pp. 235-249). Cumaná, Venezuela: Univ. Oriente.

Gaspar, M. B., Chicharo, L.M., Vasconcelos, P., García, A., Santos, A. R. \& Monteiro, C. C. (2002). Depth segregation phenomenon in Donax trunculus (Bivalvia: Donacidae) populations of the Algarve coast (southern Portugal). Sci. Mar., 66, 111-121.

Gil, G. M. \& Thomé, J. W. (2000a). Estudo do crescimento em comprimento de Donax hanleyanus Philippi, 1847 (Mollusca, Bivalvia, Donacidae). Biociências, 8, 163-175.

Gil, G. M. \& Thomé, J. W. (2000b). Morfometria da concha em Donax hanleyanus Philippi, 1847 (Mollusca, Bivalvia, Donacidae) e sua relação com as zonas de praia. Biol. Leopol., 22, 161-170.

Gil, G. M. \& Thomé, J. W. (2001). Influência de variáveis ambientais sobre uma população de Donax hanleyanus Philippi, 1847 (Mollusca, Bivalvia, Donacidae). Biotemas, 14, 109-126.
Gil, G. M. \& Thomé, J. W. (2004). Descrição do ciclo reprodutivo de Donax hanleyanus (Bivalvia, Donacidae) no sul do Brasil. Iheringia, 94, 271-276.

Leber, K. M. (1982). Seasonality of macroinvertebrates on a temperature, high wave, energy sandy beach. Bull. Mar. Sci., 32, 86-98.

Le Moal, Y. (1993). Variabilité spatiotemporelle interannuelle des populations de Donax, en Baie de Douarnenez. Bull. Ecol., 24, 75-77.

Loesch, H. C. (1957). Studies of the ecology of two species of Donax on Mustang Island, Texas. Publications of the Institute of Marine Sciences of the University of Texas, 4, 201-227.

Marcano, J. S., Prieto, A., Lárez, A. \& Salazar, H. (2003). Crecimiento de Donax denticulatus (Linné 1758) (Bivalvia: Donacidae) en la ensenada La Guardia, isla de Margarita, Venezuela. Zoot. Trop., 21, 237-259.

Mazé, R. A. \& Laborda, A. J. (1988). Aspectos de la dinámica de población de Donax trunculus (Linnaeus, 1758) (Bivalvia: Donacidae) en la ría de El Barquero (Lugo, NO España). Inves. Pesq., 52, 299-312.

McLachlan, A. (1990). Dissipative beaches and macrofauna communities on exposed intertidal sands. J. Coast. Res., 6 (1), 57-72.

McLachlan, A., Wooldridge, T. \& Van Der Horst, G. (1979). Tidal movements of the macrofauna on an exposed sandy beach in South Africa. J. Zool., 188, 433-442.

McLachlan, A., Dugan, J. E., Defeo, O., Ansell, A. D., Hubbard, D. M., Jaramillo, E. \& Penchaszadeh, P. E. (1996). Beach clam fisheries. Oceanograp. Mar. Biol. Rev., 34, 163-232. 
Mikkelsen, P. S. (1981). A comparison of two Florida populations of the coquina clam, Donax variabilis Say, 1822. I. Intertidal density, distribution and migration. Veliger, 23, 230-239.

Ocaña, F. A. \& Fernández, A. (en preparación). Uso de la Coquina (Donax striatus) en Gibara, Holguín, Cuba.

Riascos, J. M. \& Urban, H. J. (2002). Dinámica poblacional de Donax dentifer (Veneroida: Donacidae) en Bahía Málaga, pacífico colombiano durante el fenómeno "El Niño" 1997/1998. Rev. Biol. Trop., 50, 1113-1123.

Rocha-Barreira, C. A., Batista, W. F., de Oliveira Monteiro, D. \& Franklin-Junior, W. (2002). Aspectos da estructura populacional de Donax striatus (Linnaeus, 1758) (Mollusca: Donacidae) na Praia do Futuro, Fortaleza-CE. Arqu. Ciên. Mar., 35, 51-55.

Sastre, M.P. (1984). Relationships between environmental factors and Donax denticulatus populations in Puerto Rico. Estuar. Coast. Sci., 19, 217-230.

Schoeman, D. S., Wheeler, M. \& Wait, M. (2003). The relative accuracy of standard estimators for macrofaunal abundance and species richness derived from selected intertidal transect designs used to sample exposed sandy beaches. Estuar. Coast. Sci., 58, 5-16.

Soares, A. G., McLachlan, A. \& Schlacher, T. A. (1996). Disturbance effects of stranded kelp on populations of the sandy beach bivalve Donax serra (Röding). J. Exp. Mar. Biol. Ecol., 105, 165-186.

Soares, A. G., Callahan, R. K. \& De Ruyck, A. M. C. (1998). Microevolution and phenotypic plasticity in Donax serra Röding (Bivalvia: Donacidae) on high energy sandy beaches. J. Mollus. Stud., 64, 407-421.
StatSoft, Inc. (1995). STATISTICA for Windows [Computer program manual]. Tulsa, OK: StatSoft, Inc.

Tiffany, W. J. (1971). The tidal migration of Donax variabilis Say (Mollusca: Bivalvia). The Veliger, 14 (1), 82-85.

Trueman, R. D. (1971). The control of burrowing and the migratory behavior of Donax denticulatus (Bivalvia: Tellinacea). J. Zool., 165, 453-469.

Turner, J. H. \& Belding, L. D. (1957). The tidal migrations of Donax variabilis Say. Limnol. Oceanogr., 2, 120-124.

Vélez, A., Venables, B. J. \& Fitzpatrick, L. C. (1985). Growth and reproduction of the tropical beach clam Donax denticulatus (Tellinidae) in eastern Venezuela. $J$. Carib. Sci., 21 (1-2), 63-73.

Wade, B. A. (1967a). Studies on the biology of the West Indian beach clam, Donax denticulatus L. 1. Ecology. Bull. Mar. Sci., 17, 149-174.

Wade, B. A. (1967b). On taxonomy, morphology, and ecology of the beach clam, Donax striatus Linné. Bull. Mar. Sci., 17, 723-740.

Wade, B. A. (1968). Studies on the biology of the West Indian beach clam, Donax denticulatus L. 2. Life-history. Bull. Mar. Sci., 18, 876-901.

Wilson, J. G. (1999). Population dynamics and energy budget for a population of Donax variabilis (Say) on an exposed South Carolina beach. J. Exp. Mar. Biol. Ecol., 239, 61-83.

Zar, J. H. (1999). Biostatistical Analysis. (4a ${ }^{\mathrm{a}}$ ed.). Upper Saddle River: PrenticeHall Inc.

Zeichen, M. M., Agnesi, S., Mariani, A., Maccaroni, A. \& Ardizzone, G. D. (2002). Biology and Population Dynamics of Donax trunculus L. (Bivalvia: Donacidae) in the South Adriatic Coast. (Italy). Estuar. Coast. Sci., 54, 971-982. 